\title{
Opportunities for Remote Collaboration in a Social Web Search Model That Integrates Parents and Children
}

\author{
Sandra Regina Rocha Silva ${ }^{1}$, Geraldo Bonorino Xexéo ${ }^{1}$, Moacir Florentino da Silva Jr. ${ }^{2}$ \\ ${ }^{1}$ Alberto Luiz Coimbra Institute for Graduate Studies and Research in Engineering, \\ Federal University of Rio de Janeiro (UFRJ), Rio de Janeiro, Brazil \\ ${ }^{2}$ Instituto Federal de Ciência e Tecnologia do Maranhão (IFMA), Maranhão, Brazil \\ Email: profa.sandra@gmail.com, xexeo@cos.ufrj.br, moacirjunior1305@gmail.com
}

Received December 28, 2013; revised January 29, 2014; accepted February 22, 2014

Copyright (C) 2014 Sandra Regina Rocha Silva et al. This is an open access article distributed under the Creative Commons Attribution License, which permits unrestricted use, distribution, and reproduction in any medium, provided the original work is properly cited. In accordance of the Creative Commons Attribution License all Copyrights (C) 2014 are reserved for SCIRP and the owner of the intellectual property Sandra Regina Rocha Silva et al. All Copyright (C) 2014 are guarded by law and by SCIRP as a guardian.

\begin{abstract}
Traditionally, search engines are designed to support a single user working alone. However, the construction of knowledge is enriched when one adds collaboration to search tasks. We identified opportunities for remote collaboration in a Social Web search model that integrates parents and children guided by $5 \mathrm{~W}+1 \mathrm{H}$ (who, what, where, when, why, how) dimensions. Our social search model aims at improving the search process for children. We found 7 opportunities for remote collaboration on the search process, based on implicit-explicit interactions.
\end{abstract}

\section{KEYWORDS}

\section{Social Search; Collaborative Information Retrieval; Collaborative Information Seeking; Opportunities for Collaboration; Child}

\section{Introduction}

Many children today access the Internet and explore the Web from a young age. The time children aged 2 - 11 spend online increased 63\% between 2004 and 2009 [1]. Children's use of Web search engines accounts for a portion of this time increase. Unfortunately, the Web adapts slowly to the needs of children. There are many good techniques in information retrieval for adults, but still not much scientific insight on how to design search engines for children in terms of both user interfaces and underlying algorithms [2].

Search engines are traditionally designed to support a single user working alone; however, the literature describes various problems faced by children in the use of this search model: spelling, typing, query formulation, and deciphering the difficulties posed by the results [3], [4], reading and reading comprehension issues [5].

These problems exist despite the fact that children grow as digital natives, and search engines like Google offer assistance through the suggestion of key words and spelling correction mechanisms [3]. A panel of experts
[6], meeting to discuss the information behavior and needs of this new generation of users concluded that a research agenda is urgently required to study the features and preferences of this tech-savvy group that, surprisingly, lacks basic skills in information evaluation and retrieval.

Children find it difficult to grasp the information presented to them on the results page due to their cognitive abilities, the amount of information presented, and the design of the page [3]. Children were frustrated by the return of too many results and were unable to determine the most relevant and best information [7]. The large and complex volume of information to which children are exposed leads to ill-defined searches and to disorientation on the search process [8].

Evans and Chi [9] define social search as an umbrella term that describes search acts that use social interactions with others. These interactions can be explicit or implicit.

In implicit social collaboration users are not aware that their actions influenced other queries' results. Commercial search engines use implicit collaboration to improve 
the search experience (e.g.: the results clicked by other users [10]); past group query behavior is used to suggest relevant, related, search keywords [11] or even replace the user's existing query [12].

In explicitly social collaboration users are actively helping each other in their search efforts. The process of more than one person searching in collaboration with others for a shared goal is also called collaborative search [13]. The construction of knowledge is enriched when explicit collaboration is aggregated to search tasks. For example, students may need to jointly research a group homework assignment, family members might seek information about a loved one's medical condition, or friends might plan a holiday trip together [14]. As improved networking technologies and the rise of social media simplify the process of remote collaboration [13]. When groups of people search with a shared goal, they can effectively collaborate in Web search tasks, and can enjoy several advantages over solo searching, such as increased coverage of the relevant information space, higher confidence in the quality of their findings, and greater productivity due to a reduction in unnecessary redundant work [15].

Combining social information with the traditional Web search can also potentially broaden a searcher's initially narrow definition of the search topic. Combining multiple users' perspectives on how to approach a particular topic can be one of the benefits in collaborative searching, increasing the users' coverage of the information space, by combining their different information-seeking strategies and perhaps improving their individual search skills through increased exposure to the approaches other users take to investigate a topic [15].

This proposal explores the application of $5 \mathrm{~W}+1 \mathrm{H}$ (who, what, where, when, why, how) dimensions to identify opportunities for remote collaboration on search process for children. Our social search model integrates parents and children through implicit-explicit interactions.

\section{Related Work}

Bilal and Ellis [16] compared retrieved results, relevance ranking, and overlap across five non-collaborative search engines (Google, Yahoo!, Bing, Yahoo Kids!, and Ask Kids) and pointed out several limitations in these search engines projects for children.

The literature presents some search systems based in social models. The CIRS I-Spy [17] captures queries and related results for a given workgroup and uses that information to provide filtered information to users. I-Spy acts more as a collaborative filtering process than as a synchronous collaborative search. While I-Spy attempts to extend content-based filtering techniques by incorpo- rating communities, several collaborative systems have been developed by extending a traditional IR model to incorporate multiple users [18-24]. However, these social search systems were designed for the public in general, without focusing on specific child needs.

Morris, Teevan and Panovich [25] explore the pros and cons of using a social net-working tool to fill an information need, as compared with a search engine. They describe a study in which 12 subjects searched the Web while simultaneously posing a question on the same topic to their social network, and compare the results they found with each method. Although the subjects generally preferred searching, asking provided several benefits, including the delivery of customized answers and increased confidence in the validity of the search results. Their findings suggest it may be desirable to simultaneously query search engines and social tools.

People receive trusted, personalized recommendations and auxiliary social benefits when they ask questions of their friends, but using a search engine is often a more effective way to find an answer [26]. Another line of research, investigating how to adapt search engine interaction to a social context (e.g., SearchBuddies [26] responds to Facebook status message questions with algorithmic search results).

\section{5W + 1H Dimensions}

The term collaboration encompasses a variety of work configurations that vary along $5 \mathrm{~W}+1 \mathrm{H}$ dimensions, such as who forms the collaborative group and how they relate to each other (symmetric or asymmetric), what aspects of the search they need to collaborate on (process or product), where the collaborators are located (co-located or remote), when the collaboration occurs (synchronously or asynchronously), or why the group was formed [13]. The following sections present our motivations to move to $5 \mathrm{~W}+1 \mathrm{H}$ dimensions.

\subsection{The "Who" Dimension}

The who investigates the categories of people who search collaboratively. It looks at the makeup of groups, discussing typical group size and the roles group members assume and examines common relationships among collaborators, such as whether they take on symmetric or asymmetric roles in the search task. The relationships among collaborators may be either symmetric or asymmetric, depending on the degree to which the group members share an information need and depending on the role each takes on to address that need.

A symmetric collaboration is one in which the collaborators share an information need and fulfill the same roles in the search. An asymmetric collaboration is one in which the collaborators play different roles [13]. 
In our model the who dimension was set to include the groups of parents and children with asymmetric rules. The group of parents has the main functions of monitoring, searching, authorizing, and content evaluating. The group of children has further emphasized its function of browsing and searching, though children can evaluate content. The model considers the collaboration with larger groups, where groups of parents and children do not have a limitation on the number of components, with parents and children explicitly collaborating in pairs (child-child; parent-child; parent-parent).

A survey of collaborative Web search practices [14] found that $80.7 \%$ collaborated in pairs while $19.3 \%$ worked in groups of three or four members, with none reporting larger group sizes. Certain populations, such as students and families, are particularly likely to collaborate in search activities [13]. Morris and Teevan [13] identify the lack of collaborative systems that benefit from specific relationships amongst their collaborators and argue that the strength of group members' relationships can impact the success of their collaboration or the level to which they will engage in equal versus unequal roles in such an effort. Morris, Teevan, and Panovich [27] found that people were more likely to answer information requests from members of their social network whom they felt they had very close relationships with.

\subsection{The "What" Dimension}

The what dimension investigates what people's tasks are when they search collaboratively. It explores whether people need or want to collaborate in certain tasks when searching the Web. And also investigates what aspects of these tasks may benefit from collaboration [13].

The what dimension can include two collaborative search strategies: process-related collaboration (collaboration on how to find data) and product-related collaboration (where the collaboration involves exchanging the sought-after information itself) [28].

In our model, the what dimension was set to include only the search process (i.e., formulating queries, choosing results to explore). As future work, we intend to add product-related collaboration.

\subsection{The "Where" Dimension}

The where dimension investigates where the participants are located in relation to the other collaborators. The members of the group may or may not be physically at the same location and may or may not be using the same machine. When collaborators are not physically at the same place, we refer to their interaction as remote collaboration. And when all group members are physically co-present we refer to their interaction as co-located collaboration. Whether users are co-located or interact in a remote fashion, this impacts the kinds of features a collaborative search system might need to provide. Features that enable distributed control become important in co-located settings, while features supporting mutual awareness take on heightened importance in remote scenarios [13].

Some systems designed to support collaborative search have focused on co-located collaboration. Systems that support co-located collaborative Web search generally provide each group member with their own device, sometimes supplemented by a shared display [29]. CoSearch [19] facilitates co-located collaborative searching by providing a mobile phone to each group member, whereas WeSearch [29] uses a single, shared multi-touch display. Unlike these co-located configuration models that generally require the use of specific hardware, in our model the where dimension was set to encompass only remote collaboration.

\subsection{The "When" Dimension}

The when dimension investigates when the collaborators interact with each other, as search activities can occur synchronously or asynchronously. Synchronous collaboration refers to situations where all group members are working at the same time, whereas asynchronous collaboration refers to situations in which group members' efforts do not necessarily overlap temporally. Systems supporting asynchronous active collaboration amongst users with shared goals usually create a persistent artefact representing the current state of the search that can be accessed by group members at any time [13].

In our model the when dimension was set to encompass both synchronous and asynchronous collaboration.

\subsection{The "Why" Dimension}

The why dimension investigates why people work with the group members they do during collaborative search activities [13]. People work together to find information when they share an interest in the topic of the search. But people also collaborate in a search for social reasons.

There are a number of reasons as to why people collaborate: people may find it more valuable to satisfy the need with others instead of alone; people have more trust in the responses provided by friends, family, and colleagues and one way trustworthy collaborators can be identified is via long-standing relationships [27]; people may find that it provides a fun and socially-connecting experience; involving others in the search process can potentially increase the users' confidence that they have found accurate, relevant information. This increase in confidence may come from having multiple people viewing and confirming the validity of data, or from the increased coverage of the information space resulting 
from combining multiple users' search strategies [15].

A survey study [14] of information workers' collaborative search habits, though not focused on home activities found, nonetheless, that respondents provided examples of familial collaborations, including parents assisting children with homework assignments.

Another survey [27] applied to 242 users described their motivations for posing questions to their online social networks, and found as main motivations: $24.8 \%$ Trust; 21.5\% Subjective questions (e.g., a search engine can provide data but not an opinion.); 15.2\% Belief search engine would not work; $14.9 \%$ Specific audience (e.g., friends with kids, first-hand real experience.); $12.4 \%$ Connect socially; 6.6\% Answer speed; 5.4\% Context (e.g., Friends know my tastes.); 5.4\% Failed search; 5.4\% Easy; 4.1\% Answer quality (e.g., Human-vetted responses); 3.3\% No harm (e.g., No cost); $2.1 \%$ Fun; $1.7 \%$ Non-urgent. These motivations can increase our understanding of why users might choose to engage in social and collaborative search activities. The survey [27] found that $5.4 \%$ of people reported enlisting the help of their social network to find something after trying to use a search engine on their own and obtaining no results or low-relevance results. They also found that $15.2 \%$ of people believed that search engines were fundamentally incapable of answering the categories of questions they were asking, such as questions relating to breaking news or subjective opinions, and so turned to social tools to satisfy their information need without ever attempting to find the information on their own.

In our model the why dimension is associated to the need to help, guide, and police the Internet search behaviour of children, with the strengthening of the parent-child relationship and also with the monitoring of the children's activities in the Web. Children have difficulty using search engines based on a traditional search model and this is our main why.

\subsection{The "How" Dimension}

The how dimension was redefined in our model to indicate the form of social collaboration, and may take the forms of explicit social collaboration or implicit social collaboration.

In our model implicit collaboration is based on techniques of content-based filtering and collaborative filtering for predicting user preferences. Content-based filtering analyzes the content of information sources that have been rated to create a profile of the user's interests in terms of regularities in the content of the information that was rated highly. This profile may be used to rate other unseen information sources or to construct a query of a search engine. Collaborative filtering finds and recommends information sources for an individual user that have been rated highly by other users who have a pattern of ratings similar to that of the user [30].

\section{Opportunities for Collaboration}

Our social search model proposes the creation of links between group members. The links are associated with a representation and are differentiated in the following categories: links between children, where children can become friend collaborators; links between parents, where parents can become friend collaborators; links between parents and child where a father/mother can be linked to his/her child as a guardian collaborator or a member of a parents' group can be linked to a child as a friend collaborator and, in this case, it is possible for the guardian collaborator to delegate rights to this friend collaborator.

In Web-based Social Networks (WBSNs) an user is associated to two basic types of data: personal data such as name, age, and email address, and the relationship the user has with other WBSN members. These types can be modelled using the FOAF vocabulary [31] (Friend of a friend) that is an Resource Description Framework (RDF) based scheme to describe persons and their social network in a semantic way. Our social model proposes the use of the FOAF protocol to define its WBSN.

We identified 7 opportunities for remote collaboration on the search process for our social search model. Figure 1 shows the opportunities for collaboration that are described in sequence.

- Explicit synchronous remote collaboration on the search process in Figure 1: between parents (A), between parent and child (B), and between children (C). These opportunities for collaboration (A, B, C) need mechanisms that enable parents (and/or children) to evaluate content and communicate with each other in real time for the construction of a common query. For these opportunities for collaboration we propose mechanisms that support consensus, shared editors for query construction, where parents and/or children can view the query being built and also change it collaboratively in real time. As real time communication mechanisms we propose instant messaging (IM), chat. To help children in the query composition process we propose the use of natural language interfaces.

- Explicit asynchronous remote collaboration on the search process in Figure 1: between parents (D); between parent and child (E); and between children (F). These opportunities for collaboration (D, E, F) need mechanisms that allow parents (and/or children) to create a persistent representation of research in the Web to facilitate the editing, evaluating, and monitoring of search tasks. For these opportunities for collaboration we propose mechanisms such as recommendation mechanism, per-user query histories, with the including of comments and evaluation for the 


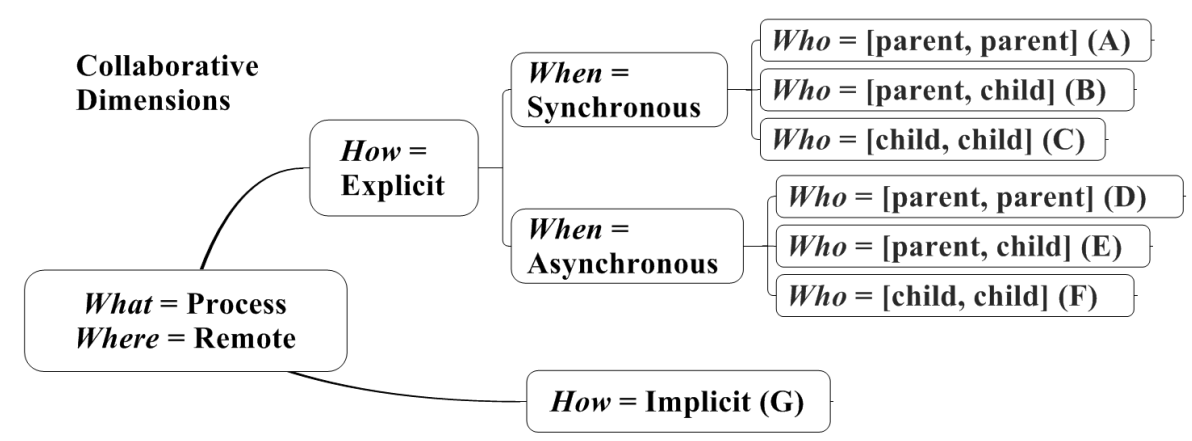

Figure 1. Opportunities for remote collaboration on search process (adapted from [32]).

query. For asynchronous communication we propose the use of email and audio email.

- Implicit collaboration on the search process in Figure 1 (G). Mechanisms of collaborative filtering and content-based filtering can be applied to the group of parents (and/or children) as mechanisms for query recommendation. Besides the creation of links between parents (and/or children) to support these forms of filtering, we propose: profiling for parents and children; per-user query histories; the association of queries in a historical to a representation; the creation of groups on a given topic; the possibility of an association of parents and/or children in a given group on a topic, the association of a representation to a group on a topic; and the association of metadata describing Web resources.

\section{Intelligent Agents}

Instant messaging, chat and other forms of computer-mediated communication (CMC) are mechanisms of explicit collaboration for our opportunities of collaboration model. Language action offers methods to analyze and model conversations such as CMC. The language action perspective of conversations is founded on the following theoretical basis: from the theory of speech act comes the assertion that, with each utterance in a conversation, an action is performed by the speaker; and these actions (or speech acts) are organized into conversations according to predefined patterns [33].

We propose the use of agents to save and treat CMC speech act profiles as a flexible mechanism to incorporate structured message passing. Twitchell et al. [34] argue that speech act profiles can be used either as patterns to classify conversations or to create visual maps of the conversations themselves. And information retrieval could benefit from the automated classification of speech acts. Filed conversations could have intent profiles attached to them that indicate the overall intention of the conversation.

Our agent model is based on an empirical study of some of the components of the agreement process using a corpus of computer-mediated dialogs and applying it to human-human collaborative dialogs in general [35]. This study [35] propose a theoretical model, and apply it to make predictions about the components of the agreement process.

\section{Conclusions}

New systems to support collaborative information seeking and retrieval should consider the constraints and desired outcomes of a wide range of contexts, including $\mathrm{K}-12$ education (i.e., primary and secondary education) [36].

Large et al. [37] reported that elementary school students often collaborate in Internet search activities, as both pedagogies of teaching in groups as the constraints of available resources for use.

Caskey [38] analyzed student (young adolescents) and parent attitudes toward school-based Internet use were explored using parent-student pairs. Positive effects of instruction were revealed to students and parents.

The search is facilitated by a participation in communities of practice, that is, groups of people who share a common goal of working practices and intellectual values. This proposal outlines as main contributions: the set of $5 \mathrm{~W}+1 \mathrm{H}$ dimensions to identify opportunities for collaboration on the search process for children; the definition of mechanisms to support these opportunities in collaboration; the integration of parents and children in a social search model; the integration of explicit and implicit social collaboration in a social search model; and the proposed use of intelligent agents to save and treat computer-mediated communication speech acts profiles as a flexible mechanism to incorporate structured messages, passing among users that explicitly collaborate in pairs.

A meta-search engine is a system that simultaneously combines results obtained from a set of search engines, re-ranking them, and presents a single ranked list to the user [39]. Our model is the base for the construction of a collaborative meta-search engine architecture for children consisting of the search engines' connection module, 
the database, explicit collaboration mechanisms and implicit collaboration mechanisms. Our model is in prototype development stage. We propose to adapt the form of evaluation applied in SearchTogether to evaluate the prototype. SearchTogether [18] was tested with a user study to evaluate how users used various tools offered in its interface and how those tools affected the act of collaboration. We also intend to complement this evaluation using an evaluation framework [40].

\section{Acknowledgements}

We should like to thank the Brazilian CAPES agency for their valuable financial support.

\section{REFERENCES}

[1] J. Nielsen, "Children's Websites: Usability Issues in Designing for Kids,” 2010.

http://www.useit.com/alertbox/children.html

[2] T. Gossen, T. Low and A. Nürnberger, "What Are the Real Differences of Children's and Adults' Web Search,” Proceedings of the 34th International ACM SIGIR Conference on Research and Development in Information Retrieval, New York, 2011, pp. 1115-1116.

[3] A. Druin, E. Foss, L. Hatley, E. Golub, M. L. Guha, J. Fails and H. Hutchinson, "How Children Search the Internet with Keyword Interfaces," Proceedings of the 8th International Conference on Interaction Design and Children, New York, 2009, pp. 89-96.

[4] S. D. Torres, D. Hiemstra and P. Serdyukov, "Query Log Analysis in the Context of Information Retrieval for Children," Proceeding of the 33rd International ACM SIGIR Conference on Research and Development in Information Retrieval, New York, 2010, pp. 847-848.

[5] J. De Belder and M.-F. Moens, "Text Simplification for Children," Proceedings of the SIGIR Workshop on Accessible Search Systems, 2010, pp. 19-26.

[6] M. L. Radford, L. S. Connaway, D. E. Agosto, L. Z. Cooper, K. Reuter and N. Zhou, "Behaviors and Preferences of Digital Natives: Informing a Research Agenda,” Proceedings of the American Society for Information Science and Technology, Vol. 44, No. 1, 2007, pp. 1-15. http://dx.doi.org/10.1002/meet.1450440133

[7] A. Large and J. Beheshti, "The Web as a Classroom Resource: Reactions from the Users," Journal of the American Society for Information Science, Vol. 51, No. 12, 2000, pp. 1069-1080. http://dx.doi.org/10.1002/1097-4571(2000)9999:9999<:: AID-ASI1017>3.0.CO;2-W

[8] S. D. Torres and I. Weber, "What and How Children Search on the Web,” CIKM, 2011, pp. 393-402.

[9] B. M. Evans and E. H. Chi, "An Elaborated Model of Social Search," Information Processing \& Management, Vol. 46, No. 6, 2010, pp. 656-678. http://dx.doi.org/10.1016/j.ipm.2009.10.012

[10] T. Joachims, "Optimizing Search Engines Using ClickThrough Data," Proceedings of the 8th ACM SIGKDD
International Conference on Knowledge Discovery and Data Mining, New York, 2002, pp. 133-142.

[11] H. Cao, D. Jiang, J. Pei, Q. He, Z. Liao, E. Chen and H. $\mathrm{Li}$, "Context-Aware Query Suggestion by Mining ClickThrough and Session Data," Proceedings of the 14th ACM SIGKDD International Conference on Knowledge Discovery and Data Mining, New York, 2008, pp. 875883. http://dx.doi.org/10.1145/1401890.1401995

[12] R. Jones, B. Rey, O. Madani and W. Greiner, "Generating Query Substitutions," Proceedings of the 15th International Conference on World Wide Web, Edinburgh, 2006, pp. 387-396. http://dx.doi.org/10.1145/1135777.1135835

[13] M. R. Morris and J. Teevan, "Collaborative Search: Who, What, Where, When, Why, and How," Morgan and Claypool Publishers, 2010.

[14] M. R. Morris, “A Survey of Collaborative Web Search Practices," Proceedings of the 26th Annual SIGCHI Conference on Human Factors in Computing Systems, Florence, 2008, pp. 1657-1660.

[15] M. R. Morris, "Interfaces for Collaborative Exploratory Web Search : Motivations and Directions for Multi-User Designs," CHI 2007 Workshop on Exploratory Search and HCI, 2007, pp. 9-12.

[16] D. Bilal and R. Ellis, "Evaluating Leading Web Search Engines on Children's Queries," Proceedings of the 14th International Conference on Human-Computer Interaction: Users and Applications, Volume Part IV, Berlin, 2011, pp. 549-558.

[17] J. Freyne, B. Smyth, M. Coyle, E. Balfe and P. Briggs, "Further Experiments on Collaborative Ranking in Community-Based Web Search," Artificial Intelligence Review, Vol. 21, No. 3-4, 2004, pp. 229-252.

http://dx.doi.org/10.1023/B:AIRE.0000036257.77059.40

[18] M. R. Morris and E. Horvitz, "SearchTogether: An Interface for Collaborative Web Search," Proceedings of the 20th Annual ACM Symposium on User Interface Software and Technology, New York, 2007, pp. 3-12. http://dx.doi.org/10.1145/1294211.1294215

[19] S. Amershi and M. R. Morris, "CoSearch: A System for Co-Located Collaborative Web Search," Proceedings of the 26th Annual SIGCHI Conference on Human Factors in Computing Systems, New York, 2008, pp. 1647-1656.

[20] M. Morris and E. Horvitz, "S3: Storable, Shareable Search," Human-Computer Interaction-INTERACT, 2007, pp. 120123.

[21] R. Krishnappa, "Multi-User Search Engine (MUSE): Supporting Collaborative Information Seeking and Retrieval," University of Missouri, 2005.

[22] S. A. Paul and M. R. Morris, "CoSense: Enhancing Sensemaking for Collaborative Web Search," Proceedings of CHI, 2009, pp. 1771-1780.

[23] C. Shah, "Coagmento: A Case Study in Designing a User-Centric Collaborative Information Seeking System," Systems Science and Collaborative Information Systems: Theories, Practices and New Research, IGI Global, 2012, pp. 242-257.

[24] M. B. Twidale and D. M. Nichols, "Collaborative Browsing and Visualisation of the Search Process,” Proceedings 
of ELVIRA-96, Milton Keynes, 1996, p. 48.

[25] M. Morris, T. Jaime and K. Panovich, “A Comparison of Information Seeking Using Search Engines and Social Networks," Presented at the Proceedings of 4th International AAAI Conference on Weblogs and Social Media, 2010, pp. 291-294.

[26] B. Hecht, T. Jaime, M. Morris and D. Liebling, "SearchBuddies: Bringing Search Engines into the Conversation," International AAAI Conference on Weblogs and Social Media, Vol. 12, 2012, pp. 138-145.

[27] M. R. Morris, J. Teevan and K. Panovich, "What Do People Ask Their Social Networks, and Why?: A Survey Study of Status Message Q\&A Behavior,” Proceedings of the 28th International Conference on Human Factors in Computing Systems, 2010, pp. 1739-1748.

[28] M. B. Twidale, D. M. Nichols and C. D. Paice, "Browsing Is a Collaborative Process,” Information Processing \& Management, Vol. 33, No. 6, 1996, pp. 761-783. http://dx.doi.org/10.1016/S0306-4573(97)00040-X

[29] M. R. Morris, J. Lombardo and D. Wigdor, "WeSearch: Supporting Collaborative Search and Sensemaking on a Tabletop Display,” Proceedings of CSCW, 2010, pp. 401410.

[30] X. Su and T. M. Khoshgoftaar, "A Survey of Collaborative Filtering Techniques," Advances in Artificial Intelligence, Vol. 2009, 2009, p. 4. http://dx.doi.org/10.1155/2009/421425

[31] D. Brickley and L. Miller, "FOAF Vocabulary Specification 0.97,” Namespace Document, 2010.

[32] S. R. R. Silva, G. B. Xexéo and M. F. da S. Junior, "Family Search Framework: Uma Arquitetura de Identificação de Oportunidades de Colaboração Para Crianças no Processo da Busca," Proceedings of I Brazilian Workshop on Social Network Analysis and Mining (BraSNAM), Curitiba, 2012.

[33] P. Goldkuhl, "Conversational Analysis as a Theoretical Foundation for Language Action Approaches?” Proceed- ings of the 8th International Working Conference on the Language-Action Perspective on Communication Modelling (LAP), Tilburg, 2003.

[34] D. P. Twitchell, M. Adkins, J. F. Nunamaker and J. K. Burgoon, "Using Speech Act Theory to Model Conversations for Automated Classification and Retrieval," Proceedings of the International Working Conference Language Action Perspective, New Brunswick, 2004, pp. 121-130.

[35] B. D. Eugenio, P. W. Jordan, R. H. Thomason and J. D. Moore, "The Agreement Process: An Empirical Investigation of Human-Human Computer-Mediated Collaborative Dialogues," International Journal of Human-Computer Studies, Vol. 53, No. 6, 2000, pp. 1017-1076. http://dx.doi.org/10.1006/ijhc.2000.0428

[36] E. M. Meyers, "Losses and Gains in Collaborative Search: Insights from the Middle School Classroom,” GROUP'10, 7-12 November 2010, Sanibel Island, 2010.

[37] A. Large, J. Beheshti and T. Rahman, "Gender Differences in Collaborative Web Searching Behavior: An Elementary School Study," Information Processing \& Management, Vol. 38, No. 3, 2002, pp. 427-443. http://dx.doi.org/10.1016/S0306-4573(01)00034-6

[38] M. M. Caskey, "Using Parent-Student Pairs for Internet Instruction,” Journal of Research on Technology in Education, Vol. 34, No. 3, 2002, pp. 304-317.

[39] J. A. Aslam and M. Montague, "Models for Metasearch," Proceedings of the 24th Annual International ACM SIGIR Conference on Research and Development in Information Retrieval, New Orleans, 2001, pp. 276-284.

[40] M. L. Wilson and M. C. Schraefel, "Evaluating Collaborative Information-Seeking Interfaces with a Search-Oriented Inspection Method and Re-Framed Information Seeking Theory,” Information Processing \& Management, Vol. 46, No. 6, 2010, pp. 718-732. http://dx.doi.org/10.1016/j.ipm.2009.10.001 